EPJ Web of Conferences 59, 05002 (2013)

DOI: $10.1051 /$ epjconf/20135905002

(C) Owned by the authors, published by EDP Sciences, 2013

\title{
Influence of the number of cones on speckle patterns in the Laser MegaJoule configuration
}

\author{
A. Le Cain ${ }^{1}$, G. Riazuelo ${ }^{2, a}$ and J.M. Sajer ${ }^{1}$ \\ ${ }^{1}$ CEA DAM CESTA, BP. 2, 33114 Le Barp, France \\ 2 CEA, DAM, DIF, 91297, Arpajon, France
}

\begin{abstract}
This paper investigates statistical properties of hot spots when speckle patterns are generated by the superimposition of multiple laser beams in a 2 cone and a 3 cone Laser MegaJoule configuration in the zone where all the beams overlap. Three different cases of polarizations are investigated: P polarization, S polarization and the case of Double Polarization Smoothing (DPS). It is found that the sizes of the speckles depend on the choice of the polarization and that DPS seems to be the best option in both configurations. It is shown that the longitudinal radius of the hot spots in a 2 cone configuration is bigger than in a 3 cone configuration.
\end{abstract}

\section{INTRODUCTION}

Irradiation uniformity is a key issue for multiple laser beam facilities such as the National Ignition Facility (NIF) in the US [1] or the Laser Megajoule (LMJ) in France [2]. A high degree of uniformity of irradiation is indeed an important requirement for the success of Inertial Confinement Fusion (ICF) because it can minimize Laser-Plasma Instabilities (LPI) such as filamentation, stimulated Brillouin scattering or stimulated Raman scattering as well as hydrodynamic instabilities.

In order to reach this uniformity, a lot of mobile hot spots (or speckles) will be generated. More precisely, Smoothing by Spectral Dispersion (SSD) [3-5] involves an irradiation of the target with a time varying speckle pattern. The coherence time is strongly reduced and the time integrated intensity has a relatively smoothed profile. The illumination on the target can then be considered as nearly uniform.

In this paper, we investigate the effect of the polarization configuration and of the number of cones on hot spot properties in the LMJ configuration in the zone where all the beams overlap. This is especially important since it has been recently shown in NIF experiments that non linear mechanisms that can take place in this zone such as energy transfer from one quad to another or multi-beam effects on backscatter could alter symetry [6] or energy coupling to the target [7]. Several choices for the polarization are possible in the context of LMJ. Three of them will be compared in this paper. In the first two cases, laser beams have the same local polarization in each quadruplet (P polarization or S polarization). In the third one (DPS [4],[8]), the electric field is splitted among two orthogonal polarizations in each quadruplet, which creates two independent speckle patterns which instantaneously interfere in the focal plane, even far from the zone where all the beams overlap.

We first remind analytical estimates for the speckle sizes and the statistical properties of the speckles in multiple beams configuration [9]. Then, we apply these formula to the case of a 2 cone and a 3 cone configuration for a LMJ irradiation scheme in these three cases of polarization.

\footnotetext{
ae-mail: gilles.riazuelo@cea.fr
}

This is an Open Access article distributed under the terms of the Creative Commons Attribution License 2.0, which permits unrestricted use, distribution, and reproduction in any medium, provided the original work is properly cited. 


\section{FORMULATION}

We consider the propagation of $\mathrm{N}$ independent speckle patterns. Let $A_{t o t}(X, Y, Z)$ result from the addition of these $\mathrm{N}$ independent speckle patterns: $A_{1} A_{2}, . . A_{N}$ :

$$
\mathbf{A}_{\text {tot }}(X, Y, Z)=\sum_{k=1}^{N} A_{k}(X, Y, Z) \text {. }
$$

The individual field components $A_{k}$ are speckle patterns with zero-mean complex Gaussian random variables [10]. That means that $A_{k}$ is the superimposition of two real-valued Gaussian fields: the real part $A_{k}^{R}$ and the imaginary part $A_{k}^{I}$ of the field with the same statistics and variances $\frac{I_{0}}{2}$, where $I_{0}$ is the average intensity of one laser beam. The different fields, $\mathbf{A}_{\mathbf{1}}, \mathbf{A}_{\mathbf{2}}, . ., \mathbf{A}_{\mathbf{N}}$, can be written:

$$
\mathbf{A}_{\mathbf{k}}=A_{k} \cos \left(\alpha_{k}\right) \mathbf{e}_{\mathbf{x}_{\mathbf{k}}}+A_{k} \sin \left(\alpha_{k}\right) \mathbf{e}_{\mathbf{y}_{\mathbf{k}}}
$$

where $\left(\mathbf{e}_{\mathbf{x}_{\mathbf{k}}}, \mathbf{e}_{\mathbf{y}_{\mathbf{k}}}, \mathbf{e}_{\mathbf{z}_{\mathbf{k}}}\right)$ is local axis-system associated to the propagation of the $k^{\text {th }}$ field.

Remark: The cases $\alpha_{k}=0$ and $\alpha_{k}=\frac{\pi}{2}$ for all the laser beams correspond to $\mathrm{P}$ and $\mathrm{S}$ polarization (respectively). In the case of DPS, two beams of the quadruplet disposed on a diagonal have the same local polarization $\alpha_{k}=0$, and the two other beams have the polarization corresponding to $\alpha_{k}=\frac{\pi}{2}$. Equation (1) can be rewritten:

$$
\mathbf{A}=\sum_{k=1}^{N} A_{k} \beta_{k} \mathbf{e}_{\mathbf{X}}+\sum_{k=1}^{N} A_{k} \lambda_{k} \mathbf{e}_{\mathbf{Y}}+\sum_{k=1}^{N} A_{k} \gamma_{k} \mathbf{e}_{\mathbf{Z}}
$$

where:

$$
\begin{aligned}
\beta_{k} & =\cos \left(\alpha_{k}\right) \cos \left(\theta_{k}\right) \cos \left(\phi_{k}\right)-\sin \left(\alpha_{k}\right) \sin \left(\phi_{k}\right), \\
\lambda_{k} & =\cos \left(\alpha_{k}\right) \cos \left(\theta_{k}\right) \sin \left(\phi_{k}\right)+\sin \left(\alpha_{k}\right) \cos \left(\phi_{k}\right), \\
\gamma_{k} & =-\cos \left(\alpha_{k}\right) \sin \left(\theta_{k}\right) .
\end{aligned}
$$

We denote $c_{k l}=\beta_{k} \beta_{l}+\lambda_{k} \lambda_{l}+\gamma_{k} \gamma_{l}$. The two next paragraphs are devoted to the study of the statistics of the hot spots of a speckle generated by multiple laser beams focused on the focal volume by lenses of focal length $\mathrm{f}$. We introduce the laser wavelength $\lambda_{0}$.

\subsection{Size of the hot spots}

In this study we shall consider speckle generated by phase plates mathematically described by $\mathrm{N}$ independent random variables which obey uniform distributions over $[0,2 \pi][11,12]$. Thanks to the simple kriging estimate, if the peak intensity $I(0)$ of the hot spot is far above the mean intensity $I_{0_{T}}$ of the total intensity, then the size of the hot spots can be approximated by [9]:

$$
\frac{I_{0}^{2}}{2} \alpha=\sum_{k=1}^{N} \sum_{l=1}^{N} \Gamma_{A_{k}}(\rho) \overline{\Gamma_{A_{l}}(\rho)} c_{k l}^{2}
$$

where $\overline{\Gamma_{A_{l}}}$ is the conjugate complex of $\Gamma_{A_{l}}$ and $\alpha=\sum_{k=1}^{N} \sum_{l=1}^{N} c_{k l}^{2}$. The Full Width at Half Maximum (FWHM) of the intensity profile, in the $\mathrm{X}, \mathrm{Y}$ and $\mathrm{Z}$ directions $\left(\rho_{X}, \rho_{Y}, \rho_{Z}\right)$ satisfies

$$
\left(\begin{array}{c}
\rho_{X}^{-2} \\
\rho_{Y}^{-2} \\
\rho_{Z}^{-2}
\end{array}\right)=\frac{1}{0.92^{2} \alpha} \sum_{k=1}^{N} \sum_{l=1}^{N} c_{k l}^{2}\left[A_{k}\left(\begin{array}{c}
\rho_{0}^{-2} \\
\rho_{0}^{-2} \\
\rho_{z}^{-2}
\end{array}\right)+\frac{3}{2 \pi^{2}}\left(k_{0}-\frac{5.5 \pi}{6 \rho_{z}}\right)^{2} B_{k l}\right]
$$




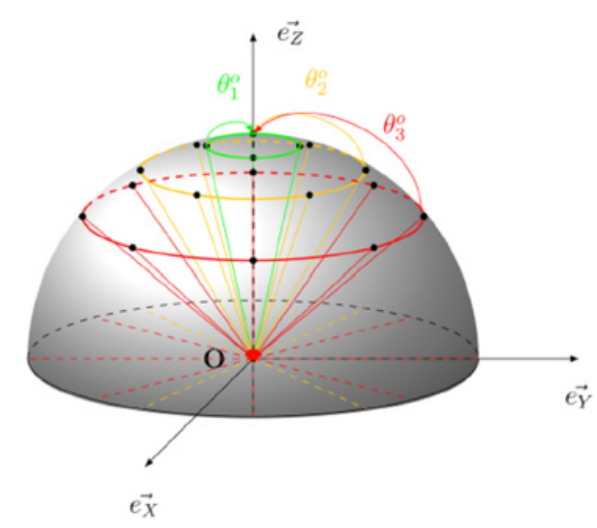

Figure 1. Here $n=3$ with $N_{1}=4$ laser beams on a sphere with angle $\theta_{1}^{\circ}, N_{2}=6$ laser beams on a sphere with angle $\theta_{2}^{\circ}$ and $N_{3}=8$ laser beams on a sphere with angle $\theta_{3}^{\circ}$. The total number of laser beams is $N=18$.

where $A_{k}$ is a transformation matrix, $B_{k l}$ is the vector representing the interference phenomenon between laser beams $k$ and $l, \rho_{0}$ is the transverse radius and $\rho_{Z}$ the longitudinal radius of a hot spot generated by only one laser beam, $\rho_{0}=\frac{\lambda_{0} f}{D}$ and $\rho_{z}=5.5 \lambda_{0}\left(\frac{f}{D}\right)^{2}$.

It appears that the sizes of the hot spots clearly depend on the configuration choices and on the coefficients $c_{k l}$, which are directly linked to the polarization of the different laser beams. This phenomenon is brought forward in the following section.

\subsection{Statistics of the total field}

We consider the total intensity, which is the superimposition of the square of six real-valued Gaussian fields $\left|A_{l}^{R}\right|^{2}$ and $\left|A_{l}^{I}\right|^{2}$ with zero-means and variances $\sigma_{l}^{2}$ for $l=1.3$.

$$
\sigma_{1}^{2}=\frac{I_{0}}{2} \sum_{k=1}^{N} \beta_{k}^{2}, \quad \sigma_{2}^{2}=\frac{I_{0}}{2} \sum_{k=1}^{N} \lambda_{k}^{2}, \quad \sigma_{3}^{2}=\frac{I_{0}}{2} \sum_{k=1}^{N} \gamma_{k}^{2} .
$$

In large laser facilities such as NIF or LMJ, the focal length $f$ is $8 \mathrm{~m}$, the focal volume is contained in $10^{-8} \mathrm{~m}^{3}$. Considering the small size of the focal volume, we make the assumption that $\frac{1}{\lambda_{0}^{2} z\left(z+\delta_{z}\right)} \sim \frac{1}{\lambda_{0}^{2} f^{2}}$.

The following results are established in the case of symmetrically distributed laser beams. $N_{j}$ laser beams are taken on the sphere as illustrated in Fig. 1. They all have the same angle $\theta_{k}=\theta_{j}^{\circ}$, the same local polarization $\alpha_{k}=\alpha_{j}^{\circ}$ and their angles $\phi_{k}$ satisfy $\phi_{k}=(k-1) \frac{2 \pi}{N_{j}} \quad k=1 . . N_{j}$ with $\sum_{j=1}^{n} N_{j}=N$.

In this configuration, and if $N_{j} \geq 3$ for $j=1 . . n$, the six real-valued Gaussian fields are mutually independent at the same point and $\sigma_{1}^{2}=\sigma_{2}^{2}$.

In the case $\sigma_{3}^{2}=0$, the density function of the total intensity is:

$$
f_{I}\left(x_{I}\right)=\frac{x_{I}}{4 \sigma_{1}^{4}} e^{-\frac{x_{I}}{2 \sigma_{1}^{2}}}, \quad x_{I} \geq 0 .
$$

In the case $\sigma_{3}^{2} \neq 0$, the density function of the total intensity is:

$$
\left.f_{I}\left(x_{I}\right)=-\frac{x_{I}}{4 \sigma_{1}^{2}\left(\sigma_{3}^{2}-\sigma_{1}^{2}\right)} e^{-\frac{x_{I}}{2 \sigma_{1}^{2}}}+\frac{\sigma_{3}^{2}}{2\left(\sigma_{3}^{2}-\sigma_{1}^{2}\right)^{2}} e^{-\frac{x_{I}}{2 \sigma_{3}^{2}}}-e^{-\frac{x_{I}}{2 \sigma_{1}^{2}}}\right), \quad x_{I} \geq 0 .
$$


Table 1. Comparison of the transverse $\rho_{X}$ and longitudinal $\rho_{Z}$ radius and the contrast $C$.

\begin{tabular}{lccc}
\hline & S polarization & P polarization & DPS \\
\hline 3 cones & $\rho_{X}=0.18 \mu \mathrm{m}$ & $\rho_{X}=0.25 \mu \mathrm{m}$ & $\rho_{X}=0.20 \mu \mathrm{m}$ \\
2 cones & $\rho_{X}=0.20 \mu \mathrm{m}$ & $\rho_{X}=0.33 \mu \mathrm{m}$ & $\rho_{X}=0.22 \mu \mathrm{m}$ \\
3 cones & $\rho_{Z}=0.68 \mu \mathrm{m}$ & $\rho_{Z}=0.73 \mu \mathrm{m}$ & $\rho_{Z}=0.69 \mu \mathrm{m}$ \\
2 cones & $\rho_{Z}=1.00 \mu \mathrm{m}$ & $\rho_{Z}=1.03 \mu \mathrm{m}$ & $\rho_{Z}=1.01 \mu \mathrm{m}$ \\
3 cones & $C=0.7071$ & $C=0.6291$ & $C=0.5828$ \\
2 cones & $C=0.7071$ & $C=0.5905$ & $C=0.5946$ \\
\hline
\end{tabular}

The contrast is given by $C^{2}=\frac{E\left[I^{2}\right]-E[I]^{2}}{E[I]^{2}}=\frac{\sum_{k=1}^{N} \sum_{l=1}^{N} c_{k l}^{2}}{N^{2}}=4 \frac{\sum_{l=1}^{3} \sigma_{l}^{4}}{N^{2}}$. In the case $\sigma_{3}^{2}=0$, the contrast is $\frac{1}{\sqrt{2}}$. The minimal one we can expect in a 3D configuration is $\frac{1}{\sqrt{3}}$. In order to estimate the growth of plasma instabilities, it is necessary to know the properties of the speckle. This is the reason why in the next section, we compare the size and the cumulative distribution function of the most intense hot spots in the case of multiple laser beams.

\section{LMJ APPLICATION}

We consider the LMJ irradiation scheme in two configurations:

1. the 3 cone configuration: with 10 quadruplets on a $33.2^{\circ}$ cone, 10 quadruplets on a $49^{\circ}$ cone and eventually 10 quadruplets on a $59.5^{\circ}$ cone. 120 laser beams are then taken on a half sphere.

2. the 2 cone configuration: with 10 quadruplets on a $33.2^{\circ}$ cone, 10 quadruplets on a $49^{\circ}$ cone. In this case, 80 laser beams are taken on a half sphere.

In what follows, we must bear in mind that the total laser energy is $50 \%$ higher in the 3 cone configuration than what it is in the 2 cone configuration.

\subsection{Size of the hot spots}

In this configuration, for the three studied cases of polarization, speckles in the main axis coordinate system have an ellipsoidal shape with radius given by equation (3). The results for the transverse and longitudinal radius are presented in table 1.

For a given geometry ( 2 cones or 3 cones), the polarization has only a slight effect on the size of the speckles. But the volume of the hot spot generated with the $\mathrm{P}$ polarization is 1.5 bigger than the volume of the speckle generated with the two other cofigurations of polarization in both geometries. But the geometry also has an influence on the size of the speckle. The transverse radii in the case of a 2 cone or a 3 cone configuration are very similar, but the longitudinal radius is larger in the case of 2 cones.

\subsection{Statistics of the total field}

We consider the complementary cumulative distribution function equations (4) and (5). We evaluate the contrast in table 1. Both the P polarization case and the DPS case reduce the number of intense speckles and make the best homogenization of the speckle pattern in the zone where all the beams overlap. The case of DPS seems to be the most efficient in both geometries: the volume of the hot spot is small and, in the same time, the probability of high intensities is reduced compared to the two other configurations (Fig. 2).

The probability of high intensity is less important in the two cone configuration than in the three cone configuration. But the longitudinal radius is at the same time bigger. So it is not possible to 


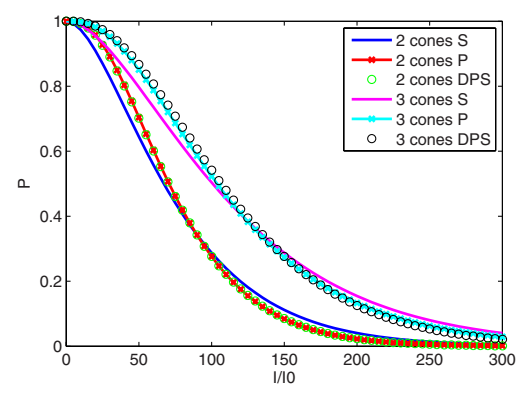

Figure 2. The complementary cumulative distribution function where $I_{0}$ is the incident intensity of one laser beam.

conclude on the best configuration to reduce laser plasma instabilities. A detailed evaluation of the global reflectivities would be necessary but this is obviously outside the scope of this paper.

\section{CONCLUSION}

The control of high intensities of smoothed beams is a crucial problem for ICF applications. Indeed, the limitation of the growth of plasma instabilities depends on the control of high intensities. In this paper we have evidenced the influence of the number of cones on the speckle patterns. It appears that a 2 cone configuration generates longer but less intense speckles. The study on the influence of the polarization configuration leads to the same conclusion in both geometries : the DPS configuration seems to be the most efficient one.

\section{References}

[1] S. W. Haan et al, Phys. plasmas 18, 051001 (2011)

[2] N. Fleurot, C. Cavailler, and J. L. Bourgade, Fusion Eng. Des. 74, 147 (2005)

[3] F. Walraet, G. Riazuelo and G. Bonnaud, Phys. Plasmas 10, 811 (2003)

[4] R. L. Berger et al, Phys. Plasmas 6, 1043 (1999)

[5] S. Skupsky and R. S. Craxton, Phys. Plasmas, 6, 2157 (1999)

[6] P. Michel et al, Phys. Plasmas, 16, 042702 (2009)

[7] R. K. Kirkwood et al, Phys. Plasmas, 18, 056311 (2011)

[8] E. Lefebvre et al, Phys. Plasmas, 5, 2701 (1998)

[9] A. Le Cain, G. Riazuelo and J.M. Sajer, Phys. Plasmas, 18, 082711 (2011)

[10] J.W. Goodman, Laser Speckle and Related Phenomena (Springer-Verlag, Heidelberg 1975) 9-75

[11] Harvey A. Rose and D. F. DuBois, Phys. Fluids B, 5, 590 (1993)

[12] J. Garnier, L. Videau, Phys. Plasmas, 8, 4914 (2001) 